\title{
Serologic responses to the PfEMP1 DBL-CIDR head structure may be a better indicator of malaria exposure than those to the DBL-a tag
}

\author{
Emily M. Stucke ${ }^{1}$, Amadou Niangaly², Andrea A. Berry ${ }^{1}$, Jason A. Bailey ${ }^{3}$, Drissa Coulibaly ${ }^{2}$, Amed Ouattara $^{1}$, \\ Kirsten E. Lyke', Matthew B. Laurens' ${ }^{1}$, Antoine Dara², Matthew Adams' ${ }^{1}$, Jozelyn Pablo ${ }^{4}$, Algis Jasinskas ${ }^{4}$, \\ Rie Nakajima ${ }^{4}$, Albert E. Zhou', Sonia Agrawal', DeAnna J. Friedman-Klabanoff', Shannon Takala-Harrison', \\ Bourema Kouriba ${ }^{2}$, Abdoulaye K. Kone ${ }^{2}$, J. Alexandra Rowe ${ }^{5}$, Ogobara K. Doumbo ${ }^{2 \wedge}$, Philip L. Felgner ${ }^{4}$, \\ Mahamadou A. Thera ${ }^{2}$, Christopher V. Plowe ${ }^{6}$ and Mark A. Travassos ${ }^{1 *}$ (i)
}

\begin{abstract}
Background: Plasmodium falciparum erythrocyte membrane protein-1 (PFEMP1) antigens play a critical role in host immune evasion. Serologic responses to these antigens have been associated with protection from clinical malaria, suggesting that antibodies to PfEMP1 antigens may contribute to natural immunity. The first N-terminal constitutive domain in a PfEMP1 is the Duffy binding-like alpha (DBL-a) domain, which contains a 300 to 400 base pair region unique to each particular protein (the DBL-a "tag"). This DBL-a tag has been used as a marker of PfEMP1 diversity and serologic responses in malaria-exposed populations. In this study, using sera from a malaria-endemic region, responses to DBL-a tags were compared to responses to the corresponding entire DBL-a domain (or "parent" domain) coupled with the succeeding cysteine-rich interdomain region (CIDR).

Methods: A protein microarray populated with DBL-a tags, the parent DBL-CIDR head structures, and downstream PfEMP1 protein fragments was probed with sera from Malian children (aged 1 to 6 years) and adults from the control arms of apical membrane antigen 1 (AMA1) vaccine clinical trials before and during a malaria transmission season. Serological responses to the DBL-a tag and the DBL-CIDR head structure were measured and compared in children and adults, and throughout the season.
\end{abstract}

Results: Malian serologic responses to a PfEMP1's DBL-a tag region did not correlate with seasonal malaria exposure, or with responses to the parent DBL-CIDR head structure in either children or adults. Parent DBL-CIDR head structures were better indicators of malaria exposure.

Conclusions: Larger PfEMP1 domains may be better indicators of malaria exposure than short, variable PfEMP1 fragments such as DBL-a tags. PfEMP1 head structures that include conserved sequences appear particularly well suited for study as serologic predictors of malaria exposure.

Keywords: Malaria, Plasmodium falciparum, var genes, PfEMP1, Immunity, Seroreactivity, Microarray

\footnotetext{
*Correspondence: mtravass@som.umaryland.edu

^Ogobara K. Doumbo-Deceased

${ }^{1}$ Malaria Research Program, Center for Vaccine Development and Global

Health, University of Maryland School of Medicine, Baltimore, MD, USA

Full list of author information is available at the end of the article
} 


\section{Background}

Plasmodium falciparum erythrocyte membrane protein-1 (PfEMP1) antigens are critical to parasite sequestration, and their genetic diversity likely facilitates host immune system evasion. The var gene family encodes PfEMP1 antigens, whose extracellular binding region is made up of Duffy-binding-like (DBL) domains interspersed with cysteine-rich interdomain regions (CIDRs). A 300-400 variable nucleotide region surrounded by semi-conserved motifs in the DBL- $\alpha$ domain, the first $\mathrm{N}$-terminal var domain, acts as a unique "fingerprint" specific to individual vars. DBL- $\alpha$ "tag" sequencing facilitates analyses of both PfEMP1 genetic diversity $[1,2]$ and seroreactivity $[3,4]$.

Antibody responses to locally derived DBL- $\alpha$ tags in Papua New Guinea increased in magnitude and prevalence with age in children, suggesting that DBL- $\alpha$ tags correlate with malaria exposure and potentially the development of natural immunity to malaria [4]. In contrast, a subsequent study of seroreactivity to 3D7 strain PfEMP1 antigens found that sera from children with cerebral malaria or severe malarial anaemia did not differ in recognition of DBL- $\alpha$ tags compared to sera from matched uncomplicated malaria controls in Bandiagara, Mali, but did with respect to larger extracellular PfEMP1 fragments [5].

To identify accurate markers of malaria exposure, serologic responses to the DBL- $\alpha$ tag of a PfEMP1 and its constitutive domains were measured. Serum samples from malaria-exposed Malian children, aged 1 to 6 years, and adults were studied on a custom PfEMP1 microarray populated with DBL- $\alpha$ tags, parent DBL-CIDR head structures (i.e. the entire DBL- $\alpha$ domains encompassing the tags with the succeeding CIDR domains), and downstream PfEMP1 fragments. The hypothesis was that seroreactivity to DBL- $\alpha$ tags among malaria-exposed individuals increases with age and with cumulative $P$. falciparum exposure. In addition, seroreactivity to the DBL- $\alpha$ tag was not predicted to correlate with seroreactivity to the parent DBL-CIDR head structure, given the head structure's additional structural components and its relative sequence conservation.

\section{Methods}

Protein microarray construction followed a four-step process that included: (1) PCR amplification of each complete or partial P. falciparum open reading frame, (2) in vivo recombination cloning, (3) in vitro transcription/translation (IVTT), and (4) microarray chip printing [6]. This IVTT protein microarray platform has produced antibody responses strongly correlated with those from ELISA assays featuring purified proteins of several malaria vaccine candidate antigens [7]. The microarray included nine DBL- $\alpha$ tags that encode Group A, B, B/A, and C PfEMP1 antigens from the 3D7 reference genome; nine matched parent DBL-CIDR head structures; and 18 matched downstream PfEMP1 fragments. Each DBL- $\alpha$ tag was expressed as a single protein fragment, and the corresponding DBL-CIDR head structure was expressed as a single protein fragment.

The protein microarrays were probed with sera from 18 adults aged 18-55 years old in the control arm of a trial of an apical membrane antigen 1 (AMA1) vaccine adjuvanted to GlaxoSmithKline's AS02A (FMP2.1/AS02A) [8] and from 35 children aged 1 to 6 years in the control arm of a Phase II AMA1 vaccine (FMP2.1/AS02A) trial [9] using published methods [6]. These serum samples were collected with respect to two malaria transmission seasons in rural Mali [adults: June 2005 (pre-malaria season) and December 2005 (post malaria season); children: May 2007 (pre-malaria season) and September 2007 (peakmalaria season)]. Sera from 11 US blood donors were used as negative controls. The data were background subtracted using the mean of the no-DNA controls, and negative fluorescence intensities were zeroed.

All participants or guardians of participants provided written informed consent, and the trial was conducted under the Declaration of Helsinki. The institutional review boards of the Faculty of Medicine, Pharmacy and Dentistry, Bamako, Mali, and the University of Maryland approved the study protocol.

\section{Analysis \\ Seroprevalence}

Seroprevalence is the proportion of serum samples that recognized a protein fragment. A serum sample recognized a protein fragment if the fluorescence intensity was greater than two standard deviations above the malaria-naïve control mean for that fragment [10]. Group "recognition" of a protein fragment was defined as a pre-malaria transmission season mean fluorescence intensity for Malian children or adults that was greater than malaria-naïve controls, based on a two-sample Kolmogorov-Smirnov test as previously described [5, 11]. The McNemar's test was used to determine if the proportions of serorecognized DBL- $\alpha$ tags and corresponding DBL-CIDR head structures were significantly discordant.

\section{Seroreactivity}

Change in seroreactivity for a protein fragment between the pre- and peak season for children and pre- and posttransmission season for adults was determined with a Wilcoxon signed-rank test for matched samples. Correlation between seroreactivity of DBL- $\alpha$ tag and DBL-CIDR head structures was measured with the Spearman correlation coefficient, $r_{s}$. P-values were two-sided, and $\alpha=0.05$. 
No adjustment was made for multiple comparisons as per previous protein microarray analyses $[5,7,12]$.

\section{Results}

Fluorescence intensities to DBL- $\alpha$ tags and their parent DBL-CIDR head structures were measured using sera collected from Malian children and adults and North American malaria-naïve adult controls (Fig. 1). Overall, increased serologic responses to the DBL-CIDR domains compared to their corresponding DBL- $\alpha$ tags were observed for both children and adults.

\section{Seroprevalence}

Comparing the seroprevalence of DBL- $\alpha$ tags and DBLCIDR head structures, serorecognition of DBL- $\alpha$ tags did not consistently predict parent DBL-CIDR head structure serorecognition (Fig. 2). For two of the nine PfEMP1 antigens, a discordant proportion of paediatric sera recognized the DBL- $\alpha$ tags compared to the proportion recognizing the corresponding parent DBL-CIDR head structure (PF3D7_0632500: $\mathrm{P}<0.0001$, PF3D7_01240600: $\mathrm{P}=0.04$, McNemar's test; Fig. 2). For all nine PfEMP1 antigens, a greater proportion of adult sera recognized the parent DBL-CIDR head structure compared to the proportion recognizing the corresponding DBL- $\alpha$ tag; however, the proportion was significantly discordant for three PfEMP1 antigens (PF3D7_1100200: $\mathrm{P}=0.0009$, PF3D7_0632500: $\mathrm{P}=0.0008$, PF3D7_0808700: $\mathrm{P}=0.003$, McNemar's test; Fig. 2).
For three PfEMP1 antigens, paediatric and adult sera demonstrated equivalent DBL- $\alpha$ tag serorecognition, but the proportion of DBL-CIDR head structure serorecognition was greater in adults versus children (PF3D7_1100200: $\quad \mathrm{P}<0.05$, PF3D7_0100300: $\mathrm{P}<0.05$, PF3D7_0200100: $\mathrm{P}<0.05$, PF3D7_0808700: $\mathrm{P}<0.0001$; PF3D7_0712000: $\mathrm{P}<0.0001$; Chi square test; Fig. 2). For PF3D7_0632500, sera from a minority of children and adults recognized the DBL- $\alpha$ tag, but sera from most children and adults recognized the parent DBL-CIDR domain head structure. This proportion was higher in adults compared to children for both the DBL- $\alpha$ tag $(\mathrm{P}<0.0001$; Chi square test) and the DBL-CIDR domain head structure $(\mathrm{P}<0.001$; Chi square test $)$.

\section{Children}

As a group, Malian paediatric sera recognized two of nine DBL- $\alpha$ tags, three of nine DBL-CIDR head structures, and four of 18 downstream PfEMP1 fragments (Fig. 3). Of the three recognized parent DBL-CIDR head structures, children recognized only one corresponding DBL- $\alpha$ tag (from PF3D7_0617400). Paediatric sera recognized the DBL- $\alpha$ tag from PfEMP1 PF3D7_0712000, but neither of the larger domain fragments that represented the extracellular region. Paediatric sera recognized all three ATS fragments, but only one of 18 extracellular PfEMP1 fragments downstream of the DBL-CIDR head structure.

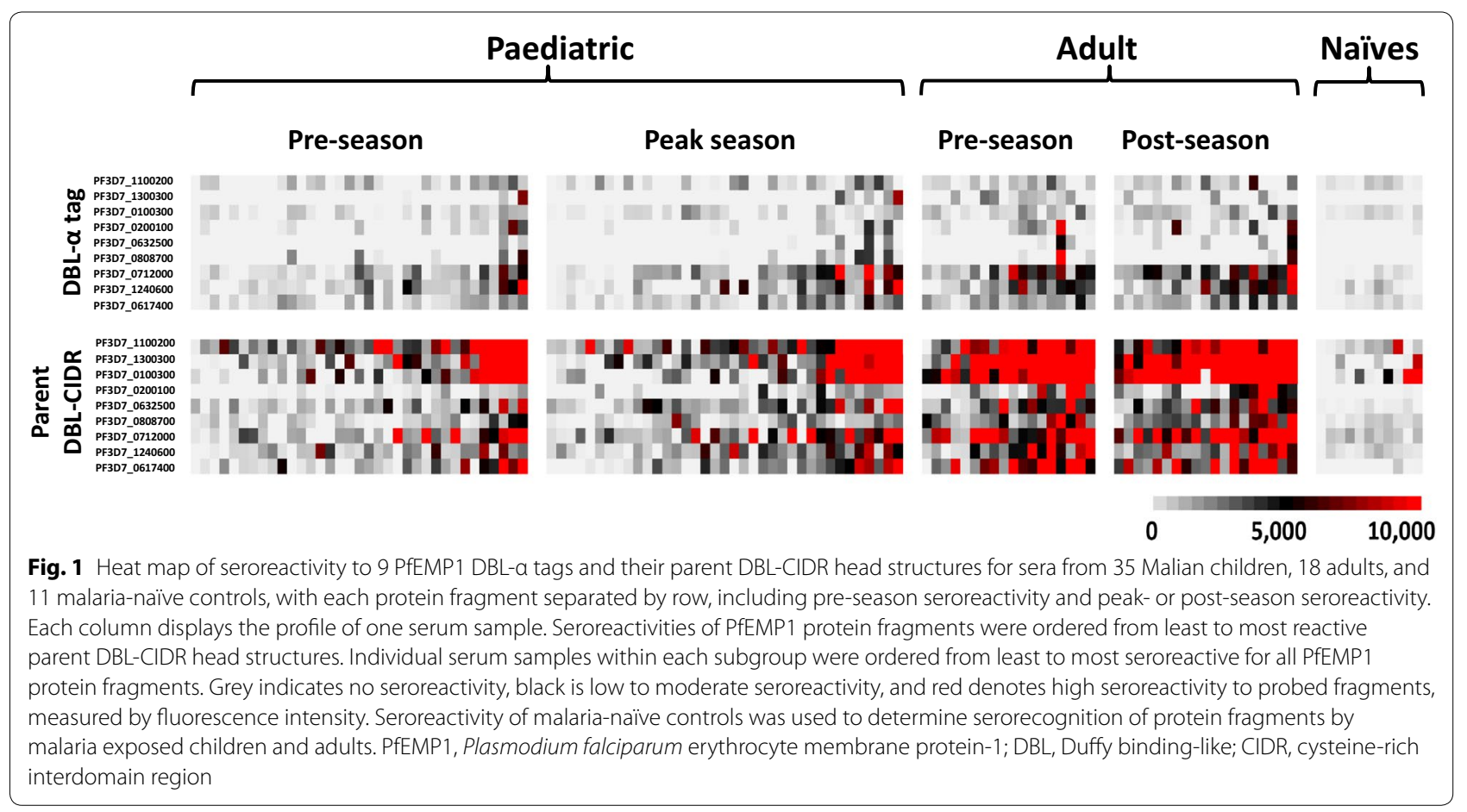




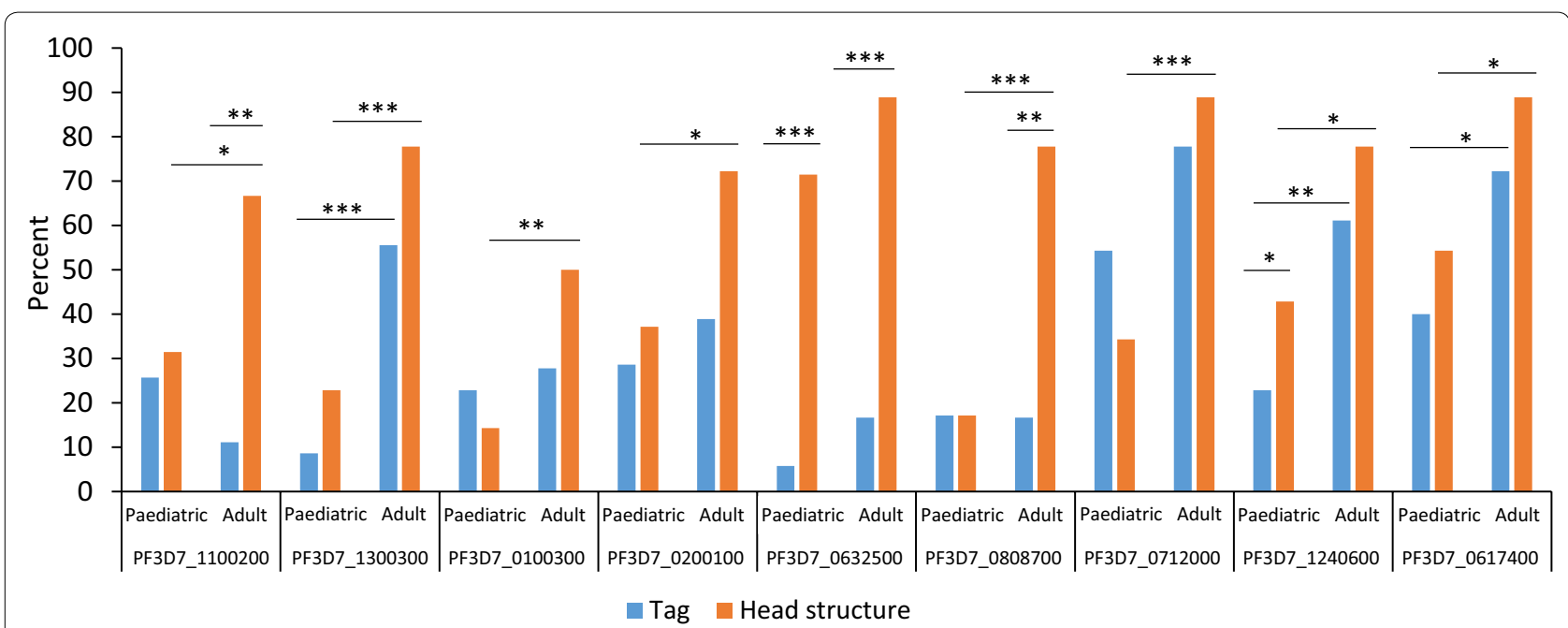

Fig. 2 Seroprevalence of DBL-a tag fragments and parent DBL-CIDR head structures recognized at pre-season for each PfEMP1 in children and adults. Paediatric sera recognized a discordant proportion of the DBL-a tag and parent DBL-CIDR head structure for two PfEMP1s, PF3D7_0632500 and PF3D7_0317400. Adult sera recognized discordant proportions of DBL-a tag and parent DBL-CIDR head structure for three PfEMP1s,

PF3D7_1 100200, PF3D7_0632500, and PF3D7_0808700. $\left({ }^{*} \mathrm{P}<0.05\right.$, ${ }^{* *} \mathrm{P}<0.01 ;{ }^{* * *} \mathrm{P}<0.001$. The McNemar's test was used to compare tags and head structures within paediatric and adults groups. For comparisons between adults and children, the Chi square test was used.) PfEMP1, Plasmodium falciparum erythrocyte membrane protein-1; DBL, Duffy binding-like; CIDR, cysteine-rich interdomain region

\section{Adults}

Malian adult sera recognized all nine of the parent DBLCIDR head structures, but only four corresponding DBL- $\alpha$ tags (Fig. 3). Adult sera recognized all 11 downstream fragments and the two ATS fragments of the four PfEMP1 antigens with serorecognized DBL- $\alpha$ tags. In total, Malian adult sera recognized 4/9 DBL- $\alpha$ tags, 9/9 parent DBL-CIDR head structures, and 18/18 downstream PfEMP1 fragments (Fig. 3).

\section{Seroreactivity \\ Children}

During a malaria transmission season, paediatric sera had increased seroreactivity to two DBL- $\alpha$ tags (PF3D7_1300300: $\quad \mathrm{P}=0.04$ and PF3D7_0712000: $\mathrm{P}=0.02$ ), but only one of the corresponding parent $\mathrm{DBL}$ CIDR head structures also had increased seroreactivity (PF3D7_0712000: $\mathrm{P}=0.01$; Fig. 3). Paediatric sera also had increased seroreactivity to two DBL-CIDR head structures (PF3D7_0632500: $\mathrm{P}=0.01$ and PF3D7_1240600: $\mathrm{P}=0.01$ ), but not to their corresponding DBL- $\alpha$ tags. Sera from children had increased seroreactivity during a malaria transmission season to only one downstream PfEMP1 fragment (PF3D7_1100200, $\mathrm{P}=0.048$; Wilcoxon signed-rank test).

\section{Adults}

For adults, seroreactivity to PfEMP1 antigens remained unchanged for all but one PfEMP1 fragment, which increased (the ATS of PF3D7_1300300: $\quad P=0.02$;
Wilcoxon signed-rank test; Fig. 3) over the course of the malaria season.

\section{Correlation of seroreactivity}

For serum samples with serorecognition of a DBL- $\alpha$ tag and/or corresponding parent DBL-CIDR head structure, correlation of the reactivities to the DBL- $\alpha$ tag (measured by fluorescence intensity) and to the corresponding DBLCIDR domain was determined. Reactivity to a DBL- $\alpha$ tag did not correlate well with reactivity to the corresponding parent DBL-CIDR head structure (Table 1). DBL- $\alpha$ tag seroreactivity significantly correlated with the parent DBL-CIDR head structure seroreactivity for only two PfEMP1 antigens (PF3D7_1100200: $r_{s}=0.81, \mathrm{P}=0.007$; PF3D7_0712000: $r_{s}=0.52, \mathrm{P}=0.002$; Table 1).

\section{Discussion}

Serologic responses to a PfEMP1's DBL- $\alpha$ tag region did not correlate with seasonal malaria exposure or with responses to the parent DBL-CIDR head structure in either adults or children from an area of Mali with intense seasonal exposure to $P$. falciparum malaria. Parent DBLCIDR head structures were better indicators of malaria exposure. Serologic prediction of malaria exposure may be better estimated by using larger PfEMP1 fragments, such as head structures that include conserved regions, as targets for serological testing. 


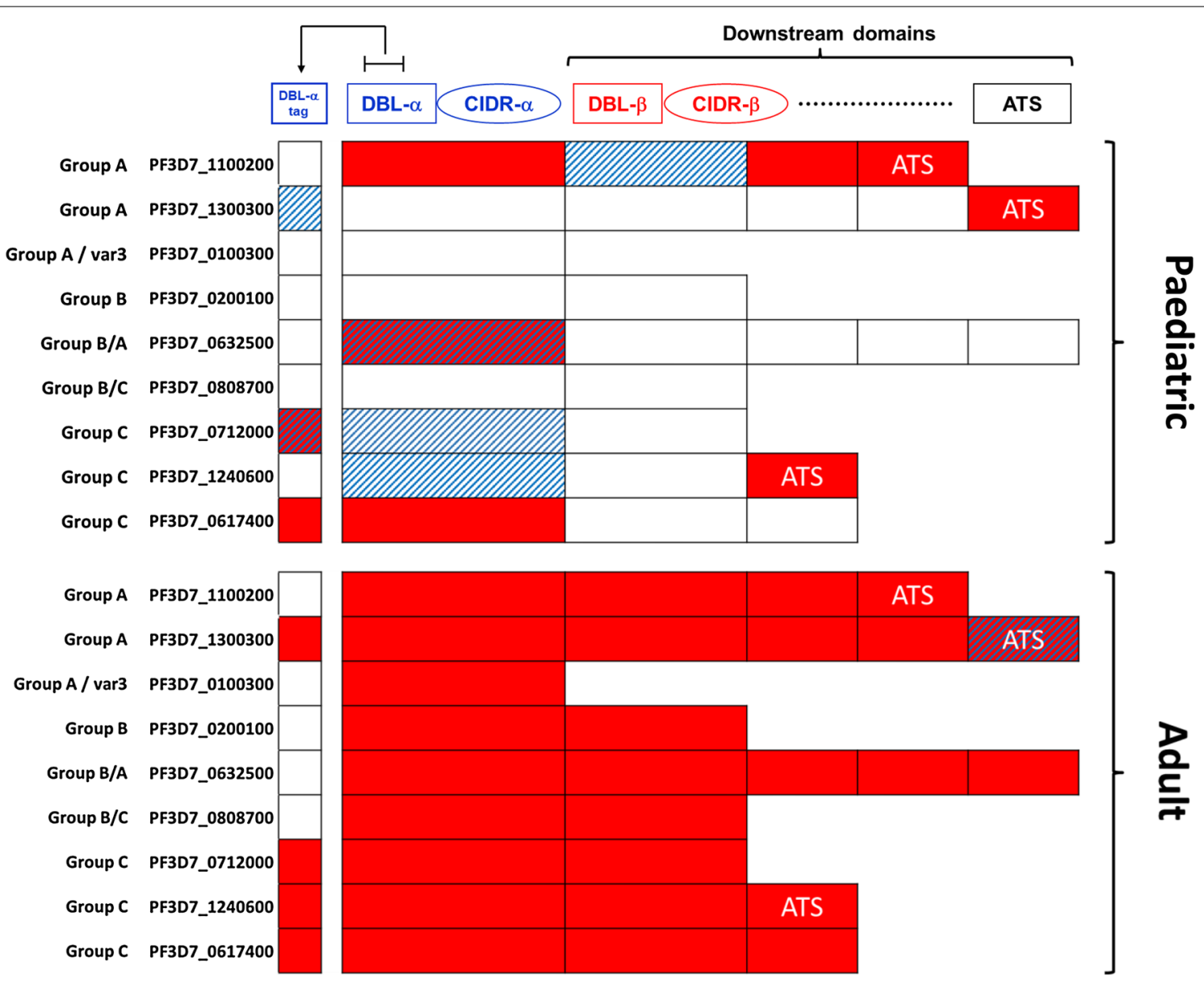

\section{Serorecognized}

Increased seroreactivity

Serorecognized \& increased seroreactivity

Fig. 3 Serorecognition and seroreactivity patterns of Malian children (top) and adults (bottom) for 36 protein fragments of nine 3D7 PfEMP1 antigens. Each DBL-a tag of a PfEMP1 antigen is the first fragment shown. Group serorecognized fragments are indicated in red, while blue stripes indicate increased seroreactivity of a fragment over a malaria season from pre-season to peak season for children and from pre-season to post-season for adults. PfEMP1, Plasmodium falciparum erythrocyte membrane protein-1; DBL, Duffy binding-like; CIDR, cysteine-rich interdomain region; ATS, acidic terminal segment

DBL- $\alpha$ tags have been used as a unique "fingerprint" reflecting PfEMP1 diversity in gene expression, serologic, and population genomics studies [4, 13-15]. These DBL- $\alpha$ tag sequences serve as a useful means of PfEMP1 classification, because each PfEMP1 contains only one DBL- $\alpha$ domain and most PfEMP1 antigens include a DBL- $\alpha$ domain. The DBL- $\alpha$ tag consists of a 300 to 400 base pair variable region flanked by conserved motifs that provide targets for degenerate primers $[16,17]$. The findings here suggest that such a unique sequence tag may not perform well as a sensitive marker for malaria exposure, potentially due to the diverse nature of the protein fragment. Seroreactivity to the parent DBL-CIDR head structure containing more conserved sequences better identified age and seasonal malaria exposure.
Table 1 Correlation of DBL-a tags and parent DBL-CIDR head structures for serorecognized PfEMP1 fragments

\begin{tabular}{lllrl}
\hline var group & PfEMP1 & $\begin{array}{l}\text { No. of serum samples } \\
\text { with serorecognition } \\
\text { of the fragment }\end{array}$ & $r_{s}$ & $P$ value \\
\hline A & PF3D7_1100200 & 11 & 0.81 & 0.007 \\
& PF3D7_1300300 & 13 & 0.42 & 0.15 \\
A/var3 & PF3D7_0100300 & 13 & 0.36 & 0.23 \\
B & PF3D7_0200100 & 17 & 0.33 & 0.19 \\
B/A & PF3D7_0632500 & 5 & -0.30 & 0.68 \\
B/C & PF3D7_0808700 & 9 & 0.58 & 0.11 \\
C & PF3D7_0712000 & 33 & 0.52 & 0.002 \\
& PF3D7_1240600 & 19 & -0.10 & 0.68 \\
& PF3D7_0617400 & 27 & 0.34 & 0.09 \\
\hline
\end{tabular}

PFEMP1 Plasmodium falciparum membrane protein-1, DBL Duffy binding-like, $C I D R$ cysteine-rich interdomain region, $r_{s}$ Spearman correlation coefficient 
These findings contrast with results from a study done in Papua New Guinea that found that serologic responses to DBL- $\alpha$ tags revealed differences in malaria exposure. One hundred twenty-three DBL- $\alpha$ tag sequences from Papua New Guinea populated a protein microarray that was used to seroprofile Papua New Guinea adults and children, revealing that children's antibody responses peaked between four and 15 years of age [4]. In this Papua New Guinea population, DBL- $\alpha$ tags served as a marker for age-related exposure to malaria. Of note, DBL- $\alpha$ tags from Papua New Guinea are less diverse than DBL- $\alpha$ tags from global populations. In a population genomic analysis of DBL- $\alpha$ tags from both Papua New Guinea and other malaria-endemic regions, the number of unique DBL- $\alpha$ tags plateaued with 30 Papua New Guinea isolates examined, but no such plateau was evident with more than 1000 DBL- $\alpha$ tag sequences from 59 global isolates [2]. Serologic responses to Papua New Guinea DBL- $\alpha$ tags may therefore be more informative than responses to DBL- $\alpha$ tags in locations with more genetically diverse DBL- $\alpha$ tags. In the current study, agerelated Malian serologic responses to DBL- $\alpha$ tags were not observed. However, we evaluated fragments from nine PfEMP1 antigens compared to the 123 evaluated from Papua New Guinea. It is possible that with a large enough pool of DBL- $\alpha$ tags on a microarray and a larger sample size, differences in age-related exposure may be discerned.

Antibody responses to particular PfEMP1 domains have been associated with protection from clinical malaria in several studies $[7,18-20]$, and may play a role in protection from severe malaria disease. A recent study of antibody responses in Malian children with severe malarial anaemia and/or cerebral malaria found gaps in immunity to particular PfEMP1 antigens compared to uncomplicated malaria controls [5]. Sera from children with severe malaria recognized fewer PfEMP1 antigens and reacted less intensely to particular PfEMP1 fragments compared to controls with uncomplicated malaria. Immunologic gaps included several DBL-CIDR domains, but only two associated DBL- $\alpha$ tags. In fact, immunologic gaps to particular DBL-CIDR head structures did not predict a gap to a particular DBL- $\alpha$ tag, and vice versa. This is consistent with the findings here that serologic responses to DBL- $\alpha$ tags were not as predictive as markers of malaria exposure compared to responses to larger PfEMP1 fragments such as head structures.

Earlier microarray studies have found that more conserved PfEMP1 fragments are markers of malaria exposure. In a study of Malian children and adults followed during the malaria season, the most differentially seroreactive fragments in adults compared to children contained the intracellular ATS domain, which is more conserved than extracellular domains [11, 21]. Similarly, in this study, paediatric sera recognized all three of the ATS domains. In Malian P. falciparum genomes, DBL- $\alpha$ and DBL- $\beta$ domains have more sequence conservation than other DBL domains [22]. Here, children recognized head structures containing DBL- $\alpha$ and DBL- $\beta$ domains while other extracellular PfEMP1 domains with lower sequence conservation remained unrecognized. Taken together, these findings suggest that when using PfEMP1 antigens as microarray markers of malaria exposure, the most conserved portions may be the most informative for malaria exposure. Such ordered serorecognition of PfEMP1 domains based on sequence conservation may also underlie the order of PfEMP1 domain recognition identified in Tanzanian children during the first 2 years of life [23, 24].

This study had some limitations. The examined DBL- $\alpha$ domains were from the reference strain 3D7, not from circulating Malian parasites in the population. In spite of this, individuals had seroreactivity to head structures and to the downstream PfEMP1 fragments, particularly Malian adults, who as a group recognized all DBL-CIDR head structures and downstream fragments. This suggests that the PfEMP1 antigens from 3D7 may represent the much larger circulating PfEMP1 population in Mali to some degree. Another limitation is the limited DBL- $\alpha$ tag population studied: DBL- $\alpha$ tags from nine 3D7 PfEMP1 antigens were examined, which represents only a small subset of the 60 3D7 PfEMP1 antigens, although at least one member of each PfEMP1 subgroup was included. This limited representation of PfEMP1 antigens could be another possible explanation for the limited serorecognition by children. It is possible that DBL- $\alpha$ tags from other 3D7 PfEMP1 antigens could have elicited more serorecognition in Malian children, but were not represented on the microarray. Whereas proteins expressed on this microarray platform have been shown to induce antibody responses similar to those from purified proteins, these proteins are produced with an Escherichia coli system and lack post-translational modifications such as glycosylation [7]. Correct folding of the PfEMP1 proteins produced by the Escherichia coli expression system has not been confirmed.

To further examine the role of conserved PfEMP1 domains in inducing serologic responses, peptide microarrays are a potentially useful tool to identify reactive epitopes. Such an approach may provide insight distinct from protein microarrays, where entire domains are included and may elicit differential seroreactivity. A protein microarray does not permit identification of the specific roles of either more conserved or more diverse regions within a protein fragment. Peptide arrays have already been used as a tool for studying epitopes in 
variant surface antigen proteins in Plasmodium falciparum. In particular, they have recently been used to pinpoint epitopes targeted by antibodies in a DBL- $\alpha$ domain that mediates rosetting [25] and epitopes within particular domains of STEVOR and RIFIN antigens reflecting malaria exposure [26]. This approach could be used to identify epitopes targeted by protective PfEMP1 antibodies and thereby inform vaccine or therapeutics design.

\section{Conclusions}

Malian serologic responses to a PfEMP1's DBL- $\alpha$ tag, a semi-conserved short fragment of the DBL- $\alpha$ domain, did not correlate with seasonal malaria exposure or responses to the parent DBL-CIDR head structure in either adults or children. Larger, more conserved PfEMP1 domains may be better indicators of malaria exposure than short, variable PfEMP1 fragments such as DBL- $\alpha$ tags. PfEMP1 head structures that include conserved sequences are thus well suited for study as serologic predictors of malaria exposure on protein microarrays. Further support for this finding would include testing a broader panel of PfEMP1 antigens, including those from non-3D7 reference strains and from endemic parasite genomes.

\section{Additional file}

Additional file 1. Sequences, classifications, and domains of the PfEMP1 antigens used to populate the custom protein microarray.

\section{Acknowledgements}

We thank Nicole Johnson, Amanda Icenroad, Ana Raquel da Costa, and Tina Williams for administrative support; the team of the Bandiagara Malaria Project for their dedication; and the community of Bandiagara, Mali.

\section{Authors' contributions}

EMS and MATr designed and analysed the experiment and drafted the manuscript. AAB, JAB, AO, MBL, AD, JP, AJ, RN, MA, ST-H, PLF, and CVP contributed to the development and printing of the microarray. JAB probed the microarrays with sera and cleaned the data. DC, KEL, AKK, MA Thera, OKD, MBL, and CVP enrolled patients, provided patient care, and collected patient data. AN, DC, BK, KEL, AKK, MATh, OKD, and CVP processed patient samples. DC, KEL, AKK, OKD, MATh, and CVP developed, funded, and coordinated the clinical studies. EMS, MATr, AN, JAB, AO, DC, AD, KEL, JAB, MBL, AEZ, SA, DF, JP, AAB, MA, ST-H, $B K, A K K, M A T h, O K D, P L F$, and CVP contributed to the approach to the data analysis. JAB and PLF contributed to the statistical analysis. All authors edited the manuscript. All authors read and approved the final manuscript.

\section{Funding}

This work was supported by an NIAID cooperative agreement (U19AI065683); NIH Grants R01Al099628 and U01Al112367; the Howard Hughes Medical Institute; an NIGMS Initiative for Maximizing Student Development Grant (2 R25-GM55036); a Passano Foundation Clinical-Investigator Award for Career Development; and a Burroughs Wellcome Fund/American Society of Tropical Medicine and Hygiene Postdoctoral Fellowship.

\section{Availability of data and materials}

Sequences used in this study are available in Additional file 1: Table S1.

\section{Ethics approval and consent to participate}

The protocol was approved by institutional review boards of the Faculty of Medicine, Pharmacy and Dentistry, Bamako, Mali and the University of Maryland, Baltimore. Written informed consent was obtained from parents or guardians of all study participants. All methods were performed in accordance with the relevant guidelines and regulations.

\section{Consent for Publication}

Not applicable.

\section{Competing interests}

The authors declare that they have no competing interests.

\section{Author details}

${ }_{1}^{1}$ Malaria Research Program, Center for Vaccine Development and Global Health, University of Maryland School of Medicine, Baltimore, MD, USA. ${ }^{2}$ Malaria Research and Training Center, University of Science, Techniques and Technologies of Bamako, Bamako, Mali. ${ }^{3}$ The EMMES Corporation, Rockville, MD, USA. ${ }^{4}$ Division of Infectious Diseases, Department of Medicine, University of California, Irvine, CA, USA. ${ }^{5}$ Centre for Immunity, Infection and Evolution, Institute of Immunology and Infection Research, School of Biological Sciences, University of Edinburgh, Edinburgh, UK. ${ }^{6}$ Duke Global Health Institute, Duke University, Durham, NC, USA.

Received: 11 March 2019 Accepted: 5 August 2019

Published online: 13 August 2019

\section{References}

1. Day KP, Artzy-Randrup Y, Tiedje KE, Rougeron V, Chen DS, RaskTS, et al. Evidence of strain structure in Plasmodium falciparum var gene repertoires in children from Gabon, West Africa. Proc Natl Acad Sci USA. 2017;114:E4103-11.

2. Barry AE, Leliwa-Sytek A, Tavul L, Imrie H, Migot-Nabias F, Brown SM, et al. Population genomics of the immune evasion (var) genes of Plasmodium falciparum. PLoS Pathog. 2007;3:e34.

3. Gitau EN, Tuju J, Karanja H, Stevenson L, Requena P, Kimani E, et al. CD4+T cell responses to the Plasmodium falciparum erythrocyte membrane protein 1 in children with mild malaria. J Immunol. 2014;192:1753-61.

4. Barry AE, Trieu A, Fowkes FJ, Pablo J, Kalantari-Dehaghi M, Jasinskas A, et al. The stability and complexity of antibody responses to the major surface antigen of Plasmodium falciparum are associated with age in a malaria endemic area. Mol Cell Proteomics. 2011;10(M111):008326.

5. Travassos MA, Niangaly A, Bailey JA, Ouattara A, Coulibaly D, Lyke KE, et al. Children with cerebral malaria or severe malarial anaemia lack immunity to distinct variant surface antigen subsets. Sci Rep. 2018;8:6281.

6. Davies DH, Liang X, Hernandez JE, Randall A, Hirst S, Mu Y, et al. Profiling the humoral immune response to infection by using proteome microarrays: high-throughput vaccine and diagnostic antigen discovery. Proc Natl Acad Sci USA. 2005;102:547-52.

7. Crompton PD, Kayala MA, Traore B, Kayentao K, Ongoiba A, Weiss GE, et al. A prospective analysis of the Ab response to Plasmodium falciparum before and after a malaria season by protein microarray. Proc Natl Acad Sci USA. 2010;107:6958-63.

8. Thera MA, Doumbo OK, Coulibaly D, Diallo DA, Kone AK, Guindo AB, et al. Safety and immunogenicity of an AMA-1 malaria vaccine in Malian adults: results of a phase 1 randomized controlled trial. PLOS ONE. 2008;3:e1465.

9. Thera MA, Doumbo OK, Coulibaly D, Laurens MB, Ouattara A, Kone AK, et al. A field trial to assess a blood-stage malaria vaccine. N Engl J Med. 2011;365:1004-13.

10. Bailey JA, Pablo J, Niangaly A, Travassos MA, Ouattara A, Coulibaly D, et al. Seroreactivity to a large panel of field-derived Plasmodium falciparum apical membrane antigen 1 and merozoite surface protein 1 variants reflects seasonal and lifetime acquired responses to malaria. Am J Trop Med Hyg. 2015;92:9-12.

11. Travassos MA, Niangaly A, Bailey JA, Ouattara A, Coulibaly D, Laurens MB, et al. Seroreactivity to Plasmodium falciparum erythrocyte membrane protein 1 intracellular domain in malaria-exposed children and adults. J Infect Dis. 2013;208:1514-9. 
12. Travassos MA, Coulibaly D, Bailey JA, Niangaly A, Adams M, Nyunt MM, et al. Differential recognition of terminal extracellular Plasmodium falciparum VAR2CSA domains by sera from multigravid, malaria-exposed Malian women. Am J Trop Med Hyg. 2015;92:1190-4.

13. Warimwe GM, Keane TM, Fegan G, Musyoki JN, Newton CR, Pain A, et al. Plasmodium falciparum var gene expression is modified by host immunity. Proc Natl Acad Sci USA. 2009;106:21801-6.

14. Warimwe GM, Recker M, Kiragu EW, Buckee CO, Wambua J, Musyoki $\mathrm{JN}$, et al. Plasmodium falciparum var gene expression homogeneity as a marker of the host-parasite relationship under different levels of naturally acquired immunity to malaria. PLoS ONE. 2013;8:e70467.

15. Kyriacou HM, Stone GN, Challis RJ, Raza A, Lyke KE, Thera MA, et al. Differential var gene transcription in Plasmodium falciparum isolates from patients with cerebral malaria compared to hyperparasitaemia. Mol Biochem Parasitol. 2006;150:211-8.

16. Taylor HM, Kyes SA, Harris D, Kriek N, Newbold Cl. A study of var gene transcription in vitro using universal var gene primers. Mol Biochem Parasitol. 2000;105:13-23.

17. Kyes S, Taylor H, Craig A, Marsh K, Newbold C. Genomic representation of var gene sequences in Plasmodium falciparum field isolates from different geographic regions. Mol Biochem Parasitol. 1997;87(2):235-8.

18. Marsh K, Otoo L, Hayes RJ, Carson DC, Greenwood BM. Antibodies to blood stage antigens of Plasmodium falciparum in rural Gambians and their relation to protection against infection. Trans R Soc Trop Med Hyg. 1989;83:293-303.

19. Chan JA, Howell KB, Reiling L, Ataide R, Mackintosh CL, Fowkes FJ, et al. Targets of antibodies against Plasmodium falciparum-infected erythrocytes in malaria immunity. J Clin Invest. 2012;122:3227-38.

20. Chan J-A, Fowkes FJI, Beeson JG. Surface antigens of Plasmodium falciparum-infected erythrocytes as immune targets and malaria vaccine candidates. Cell Mol Life Sci. 2014;71:3633-57.
21. Gardner MJ, Hall N, Fung E, White O, Berriman M, Hyman RW, et al. Genome sequence of the human malaria parasite Plasmodium falciparum. Nature. 2002;419:498-511.

22. Dara A, Drabek EF, Travassos MA, Moser KA, Delcher AL, Su Q, et al. New var reconstruction algorithm exposes high var sequence diversity in a single geographic location in Mali. Genome Med. 2017;9:30.

23. Cham GK, Turner L, Kurtis JD, Mutabingwa T, Fried M, Jensen AT, et al. Hierarchical, domain type-specific acquisition of antibodies to Plasmodium falciparum erythrocyte membrane protein 1 in Tanzanian children. Infect Immun. 2010;78:4653-9.

24. Cham GK, Turner L, Lusingu J, Vestergaard L, Mmbando BP, Kurtis JD, et al. Sequential, ordered acquisition of antibodies to Plasmodium falciparum erythrocyte membrane protein 1 domains. J Immunol. 2009;183:3356-63.

25. Angeletti D, Albrecht L, Blomqvist K, Quintana Mdel P, Akhter T, Bachle SM, et al. Plasmodium falciparum rosetting epitopes converge in the SD3loop of PfEMP1-DBL1alpha. PLoS ONE. 2012;7:e50758.

26. Zhou AE, Berry AA, Bailey JA, Pike A, Dara A, Agrawal S, et al. Antibodies to peptides in semiconserved domains of RIFINs and STEVORs correlate with malaria exposure. mSphere. 2019;4:e00097.

\section{Publisher's Note}

Springer Nature remains neutral with regard to jurisdictional claims in published maps and institutional affiliations.
Ready to submit your research? Choose BMC and benefit from:

- fast, convenient online submission

- thorough peer review by experienced researchers in your field

- rapid publication on acceptance

- support for research data, including large and complex data types

- gold Open Access which fosters wider collaboration and increased citations

- maximum visibility for your research: over $100 \mathrm{M}$ website views per year

At BMC, research is always in progress.

Learn more biomedcentral.com/submissions 